\title{
Incidencia del impuesto al valor agregado iva en las \\ utilidades de las empresas de dragado e ingeniería allmar sas
}

\section{Impact of tax to value added iva in profit of dredging and engineering company allmar sas}

Alejandro Trujillo ${ }^{1}$

\section{Resumen}

El presente artículo tiene la intención de comparar la incidencia del Impuesto sobre las ventas en las utilidades de las empresas de dragado e ingeniería calculando dicho impuesto sobre el AIU y sobre los ingresos totales durante el periodo 2014. Teniendo en cuenta las diferentes dificultades o problemas que se presentan en las empresas de Dragado e Ingeniería con relación a la determinación y/o conveniencia de aplicar o no la modalidad del AIU en la celebración de sus contratos realizados para determinar el Impuesto a las Ventas, en la medida que no se tenga claridad dentro de la realización de su operación sus utilidades van relacionadas directamente con el concepto de construcción o simplemente como un servicio. Por tanto se realiza esta investigación para identificar cuáles son los conceptos que se deben tener en cuenta para que sus actividades sean consideradas como servicios de construcción y así poder aplicar la modalidad de AUI para la liquidación del impuesto sobre las ventas.

\footnotetext{
${ }^{1}$ Contador Público AGLALA ISNN 2215-7360

2015; 6 (1): 229-251
} 
Docente en la Corporación Universitaria Rafael Núñez alejandro.trujillo@curnvirtual.edu.co CÓDIGO JEL: $\mathrm{H} 24$

Fecha de recepción: Abril de 2015 / Fecha de aceptación en forma revisada: Julio 2015

Palabras clave: IVA; impuesto; utilidades; empresas de dragado e ingeniería; servicio.

\begin{abstract}
This paper intends to compare the incidence of sales tax on company profits dredging and engineering calculating the tax on AIU and total revenues for the period 2014. Considering the various difficulties or problems that arise in business and engineering Dredging regarding the determination and / or convenience whether to apply the mode of AIU in celebration of their contracts performed to determine the Sales Tax, to the extent that there will be clarity in conducting its operation profits go directly related to the concept of building or just as a service. Therefore this research is done to identify the concepts that must be taken into account so that their activities are considered as construction services and be able to apply the AUI mode for settlement of sales tax are.
\end{abstract}

Keywords: VAT; tax; utilities; dredging and engeneering business; service

\title{
Introducción
}

El sistema tributario Colombiano ha venido evolucionando para lograr la integración entre las características de los tributos que lo conforman, particularidades y necesidades económicas del país. Es por ello, que las diversas disposiciones legales que regulan los tributos en Colombia han tenido modificaciones y reformas para hacer frente a las necesidades públicas para satisfacer a la colectividad mediante la actuación del Estado.

AGLALA ISNN 2215-7360

2015; 6 (1): 229-251 
De manera que, el Estado necesita de los tributos para poder auxiliar los gastos públicos, puesto que la evasión fiscal es un problema de cultura, y es responsabilidad del gobierno crear esa cultura en los contribuyentes. Adicionalmente, el Estado busca justificar el incremento de las alícuotas impositivas mediante la reforma de la ley que establece el Impuesto al Valor Agregado (IVA) para hacer lo propio o eliminar algunas exenciones o diluir el efecto en la población por la creación de nuevos impuestos como al patrimonio, al consumo selectivo de bienes o servicios entre otros.

El IVA es un impuesto indirecto de carácter nacional que se calcula sobre el consumo, el cual como su nombre lo indica se genera sobre el valor agregado, es decir, sobre el valor que el vendedor agrega al producto, ya que con la entrada en vigencia del decreto 1372 se estableció en sus artículos 3 y 8, dos modalidades para el cálculo del impuesto; en el caso de las empresas que se encargan del dragado no se tiene conocimiento de la aplicación sobre las ventas en tanto para el modelo de AIU como para la liquidación sobre los ingresos totales. Por tal razón, el presente artículo tiene como objetivo comparar la incidencia del Impuesto sobre las ventas en las utilidades de las empresas de dragado e ingeniería calculando dicho impuesto sobre el AIU y sobre los ingresos totales durante el periodo 2014.

\section{Antecedente Histórico de la Administración Tributaria en Colombia}

Los tributos dentro de la organización del Estado constituyen la fuente de financiación los distintos proyectos de inversión pública, garantizando además el funcionamiento del Estado, por lo que la Constitución de Colombia 1991 dispuso en el numeral 9 del artículo 95, ya que la 
misma establece los deberes de las personas en cuanto a contribuir al financiamiento de los gastos e inversiones del Estado con justicia y equidad (Constitución Política de Colombia, 1991.

De manera que, para Jacobo Pérez el Estado para cumplir sus fines ha de ejercer funciones y prestar servicios, los cuales deben provocar un beneficio hacia la comunidad y sus miembros, los cuales deben ser costeados por los miembros de la respectiva comunidad mediante una contribución señalada por el Estado o bien mediante la explotación de los bienes pertenecientes a la colectividad o al Estado... (Pérez, 2004)

Ante todo es necesario convenir que el principio de convocar a la sociedad a participar en las necesidades públicas permite comprender que es necesario para el Estado construir un sistema económico que cumpla con sus fines esenciales, apoyado por en un sistema tributario acorde con las exigencias y necesidades de los ciudadanos, como también el sistema de régimen de gobierno adoptado para este fin. Por tanto, los impuestos deben diseñarse cumpliendo finalidades de redistribución de la riqueza, con criterios de progresividad en función de la capacidad económica de los contribuyentes, suficientes para realizar las obras de infraestructura y beneficio social, además que incentiven la productividad en el sector privado para la estabilidad económica dentro del marco macroeconómico nacional y regional (Díaz y Varela, 2003).

Es importante señalar que el objetivo fundamental de los impuestos es el cubrir los gastos públicos desalentando la producción de algunos bienes, construyendo impuestos adicionales que elevan el precio de un producto; modificar la distribución de la renta, puesto que los tributos, el gasto público y las transferencias son las herramientas con que cuenta el Estado para llevar a cabo una política de redistribución del ingreso. Además, dichos impuestos llegan a ser 
proporcionales, progresivos o regresivos, según ordenación de los tributos presentes dentro sistema tributario.

Ciertamente, los grupos sociales compiten en el intento de disminuir la carga que gravita sobre ellos, puesto que un sistema tributario interrelacionado de impuestos que rige en un país determina el cumplimiento de algunos requisitos para lograr ser una estructura tributaria apta. Por tanto, un sistema tributario debe estar diseñado para:

- Alcanzar los objetivos de la política fiscal, maximizando la equidad en la asignación de los recursos de la economía, promoviendo la estabilidad y el crecimiento económico.

- Disminuir los costos del sistema, mediante la percepción y el control impositivo.

- Obtener un rendimiento fiscal idóneo, que implique conseguir una elevada recaudación que incida en la economía (Haindl, 2005).

Aunado a ello, el sistema tributario debe cumplir con características específicas, por lo que debe contar con más de un tributo, ser directos e indirectos, contar con un código organizado, poseer una Administración Tributaria tecnológicamente organizada y darle al país una cultura tributaria adecuada.

\section{Contar con más de un tributo.}

La Constitución de 1991 establece en su artículo 95, numeral 9 como responsabilidad de los miembros de la comunidad nacional, contribuir a los gastos e inversiones del estado dentro de los conceptos de justicia y equidad (Bravo, 2003). 


\section{Tributos directos e indirectos.}

Los tributos directos Estos recaen sobre los ingresos de trabajo y propiedad de las personas gravadas, afectando la capacidad de pago, de renta, patrimonio, remesas, ganancias ocasionales, cotizaciones a la Seguridad Social, entre otros). Los tributos indirectos son pagados por una persona diferente a la que afecta el pago del mismo; considerando el traspaso de la obligación tributaria del responsable ante el Estado a un tercero cuyos ingresos son afectados por el pago del tributo (DIAN, 2005).

\section{Contar con un código organizado}

Toda norma que integra el ordenamiento jurídico Colombiano debe ser concebida para el cumplimiento de los derechos, deberes y obligaciones contenidos en la misma, puesto que la ley general tributaria tiene una vocación de inclusión, regulando de esta manera un conjunto orgánico y sistemático de disposiciones que regulan la materia en general (Correa, 2002).

\section{Administración tecnológicamente organizada}

La administración tributaria en Colombia ha venido mejorando, debido al desarrollo eficaz de la tarea de fiscalización y cobro, diseñando una aplicación tecnológica como herramienta de planeación y control de la gestión de los tributos que administra. En este sentido, la Dirección de Impuestos y Aduanas Nacionales de Colombia ha adaptado un sistema de información integral (MUISCA), que le permite llevar un control eficiente sobre el sector tributario y aduanero en el país, y que los contribuyentes puedan tener un mejor servicio al momento de hacer consultas sobre sus obligaciones fiscales o de exigir derechos (Ministerio de 
Comunicaciones, 2008).

\section{País con buena cultura tributaria}

Propiciar un cambio cultural en los contribuyentes en Colombia es incorporar a toda la población a cumplir con sus obligaciones tributarias, por ello el reto del Estado es trabajar en la cultura ciudadana. Esto garantiza el pago de los tributos exigiendo además la participación de los ciudadanos y la utilización de los instrumentos que contiene la Constitución de 1991, manteniendo la eficiencia a través de la vigilancia de la gestión pública y la asignación del gasto, para la obtención de la rendición de cuentas por parte de los servidores (DIAN, 2010).

Tal como se evidencia, el desarrollo de la administración tributaria está vinculada al desarrollo del Estado, de la economía y al comportamiento cultural de la ciudadanía, puesto que las organizaciones tributarias poseían un papel más distante en cuanto al cumplimiento de sus actividades, por otro lado eran concebidas como entes de perseguir los recaudos por los impuestos administrados sin entrar considerar al contribuyente, considerándose organizaciones poco modernas. Asimismo, la administración tributaria ha tomado en consideración a sus contribuyentes dotándoles de posibilidades con lo cual puedan contar con mejores medios en cuanto a la asistencia al contribuyente, siendo éstos es pilar fundamental en la recaudación a un menor costo en cuanto a tiempo, recursos humanos y físicos que son utilizados (Ministerio de Hacienda y Crédito Público. 2010).

La administración tributaria se orienta a organizar, dirigir y controlar de los impuestos con el propósito de satisfacer las necesidades del Estado y de sus administrados, estableciendo la relación impositiva por parte de los sujetos pasivos, recaudando el importe de los mismos y 
resolver las impugnaciones efectuadas por los contribuyentes. Por otro lado, la administración posee funciones sustantivas o primarias y de apoyo o soporte para el desarrollo eficaz de esas funciones primarias (Monsalve, 2008).

Las funciones o procesos sustantivos en la cual la organización administra el cumplimiento de las obligaciones tributarias a través del recaudo de los impuestos, determinando así el control de la evasión, el fraude y el cobro ejecutivo de los montos obligados. Por tanto, del cumplimiento de las obligaciones surge el diseño de sistemas tributarios más equitativos como procesos esenciales o primarios que se dan mediante la recaudación, fiscalización, determinación/ liquidación, legal o apelaciones, cobro y asistencia al contribuyente.

Las funciones o procesos de soporte o apoyo, suplen las necesidades de recursos humanos, para el desarrollo de las funciones primarias, suministro de recursos físicos, consecución y procesamiento de la información brindada por los contribuyentes y por terceros para el control del cumplimiento de las obligaciones. De igual forma, este proceso se orienta a la planeación y control de la gestión de la administración a través de planes organizacionales y del diseño de sistemas de medición a nivel interno y externo dentro de la organización, definiendo como procesos de apoyo de apoyo la Planeación Estratégica, Recursos Humanos; Recursos Financieros; Informática Tributaria; Cooperación Internacional (Steiner y Medellín, 2014).

Es de destacar que el desarrollo de los procesos como soportes de la administración tributaria sirve de apoyo, permitido mayor agilidad y eficacia en la gestión de la información. Además, mejora la gestión de la información a través de la automatización, permitiendo producir reportes y procesos de recaudación, control y cobro, todo ello implica cambios y ventajas de la 
tecnología permitiendo contar con mayor control sobre el desarrollo del trabajo de la misma organización (Moller, 2012).

\section{Antecedentes}

La entrada en vigencia del Decreto 624 de (1989) en el art. 420 establecía que el hecho generador del Impuesto sobre las Ventas era la prestación de los servicios especificados en el artículo 476, estableciendo que los servicios excluidos del tributo eran gravados y cuáles eran excluidos del impuesto. Este mismo decreto buscaba unificar las diferentes normas en materia tributaria, según su artículo 437 señalaba que los responsables de los impuestos en cuanto a la prestación de servicios eran Quienes presten los servicios gravados, exonerando a las personas que prestaran servicios excluidos y exentos (Estatuto Tributario Decreto 624, 1989).

La entrada en vigencia de la Ley 6 de (1992) modificó el literal b) del artículo 420 donde el hecho generador del tributo era mediante la prestación de servicios en el territorio nacional, y a su vez, determina la responsabilidad del impuesto a todas las personas que presten servicios dentro del territorio colombiano son responsables del impuesto sobre las ventas según el literal c) del artículo 437 (Ley 6, 1992).

Por su parte, la Ley 6 de 1992 trajo el decreto reglamentario 1372 de (1992) estableciendo la definición de servicios para efectos del IVA como toda actividad, labor o trabajo prestado por una persona natural o jurídica, o por una sociedad de hecho, sin relación laboral con quien contrata la ejecución, que concretada en una obligación de hacer, sin importar el factor material o intelectual que p0redomine, que genera una contraprestación en dinero o en especie, 
independientemente de su denominación o forma de remuneración según el artículo 1 (Decreto Reglamentario 1372, 1992).

Asimismo, se implementó el impuesto sobre las ventas en los contratos de Construcción de bienes inmuebles que se genera sobre la parte de los ingresos correspondiente a los honorarios obtenidos por el constructor. Por su parte, el artículo 8 que en los servicios gravados con el impuesto sobre las ventas la base gravable estará conformada por la totalidad de los ingresos percibidos por el servicio, aun cuando tengas facturaciones por separada, generando dos bases gravables diferentes una que es sobre el valor correspondiente al AUI en los servicios de construcción y otra sobre la totalidad del ingreso percibido en el servicio (Decreto Reglamentario 1372, 1992).

Adicionalmente, se unificó el concepto en los contratos de construcción, urbanización y de confección de obra material de bien inmueble todos los que el contratista, directa o indirectamente, edifica, fabrica, erige o levanta las obras, edificios, construcciones para residencias o negocios, puentes, carreteras, represas, acueductos, edificaciones en general y obras inherentes a la construcción tales como: electricidad, plomería, cañería, mampostería, drenajes y todos los elementos que se incorporen a la construcción. Por tanto, no constituyen contratos de construcción las obras o bienes que puedan retirarse sin pérdida del inmueble, como divisiones internas en edificios ya terminados (IVA $\mathrm{N}^{\circ}$ 001, 2003).

\section{La inclusión del AIU en los procesos de contratación}

Para la implementación del modelo de AIU en la empresas de dragado e ingenierías se debe tener en cuenta qué tipo de actividades pueden usar esta modalidad, ya que los contratos no 
se deben al cumplimiento de una disposición legal sino a la discriminación del costo total de la obra, costos directos e indirectos, con fines de evaluación de las propuestas, verificación de su ejecución, resolver posibles discusiones que surjan en al equilibrio contractual e indemnizaciones. El AIU llamados costos indirectos del contrato y no incluye los Costos Directos, ya que aquellos que tienen relación directa con la ejecución del objeto del contrato (Concepto 017713, 2010).

Se tiene en consideración que el AIU no obedece al cumplimiento de una disposición legal, aplicándose en los contratos de construcción tal como establece el artículo 3 del Decreto 1372 de (1992), en cual el impuesto sobre las ventas se genera sobre los ingresos correspondientes a los honorarios que son obtenidos por el constructor. Cuando no se pacten honorarios el impuesto se causará sobre la remuneración del servicio que corresponda a la utilidad del constructor. Para estos efectos, en el respectivo contrato se señalará la parte correspondiente a los honorarios o utilidad, la cual en ningún caso podrá ser inferior a la que comercialmente corresponda a contratos iguales o similares (Decreto 1372, 1992).

\section{Elementos que debe tener un contrato para aplicar la modalidad del AIU}

Los elementos que se deben tener en cuenta al momento de celebrar un contrato de construcción y aplicar la modalidad del AIU está contemplado en el concepto unificado del impuesto sobre las ventas son contratos de construcción, urbanización, de confección de obra material de bien inmueble aquellos por los cuales el contratista, directa o indirectamente, edifica, fabrica, erige o levanta las obras, edificios, construcciones para residencias o negocios, puentes, 
carreteras, represas, acueductos, edificaciones en general y las obras inherentes a la construcción en sí, tales como: electricidad, plomería, cañería, mampostería, drenajes y todos los elementos que se incorporen a la construcción (IVA N 001, 2003).

Una vez analizados los elementos que conforman un contrato de construcción es importante identificar el beneficio que se obtiene al aplicar esta modalidad de liquidación como lo establece el Concepto 044743 De (2013):

a. Instituye la base especial para liquidar el IVA por parte del contratista según se contrate por honorarios;

b. Coloca que el IVA pagado por los costos y gastos es mayor valor del costo o del gasto respectivo, esto es mayor valor finalmente de la obra construida;

c. Limita el empleo del IVA descontable para el contratista constructor sólo al correspondiente a sus gastos propios;

d. Obliga a que dentro del contrato de construcción se exprese o la utilidad que el constructor espera de su contrato o los honorarios pactados;

e. Remite a contratos iguales o similares para establecer la base gravable en caso de no haber sido expresada en el contrato, tomando en cuenta las circunstancias de la obra construida (Concepto 044743, 2013).

Para poder definir si hay un beneficio o no del cálculo del AIU en la empresa de dragado hay que determinar las dos modalidades de liquidación del impuesto ya sea en los contratos de bienes inmuebles o en los servicios gravados que involucran utilización de inmuebles. De manera que, los contratos de construcción el impuesto se liquida sobre la remuneración del servicio que corresponda a la utilidad del constructor y en los servicios gravados que involucran utilización 
de inmuebles la base gravable estará conformada por la totalidad de los ingresos percibidos por el servicio, aun cuando se facturen en forma separada (Decreto 1372, 1992).

Es importante determinar que para los contratos de construcción hay una limitación en las deducciones como lo consagra artículo 3 el responsable sólo podrá solicitar impuestos descontables por los gastos directamente relacionados con los honorarios percibidos o la utilidad obtenida, que constituyeron la base gravable del impuesto; en consecuencia, en ningún caso dará derecho a descuento el impuesto sobre las ventas cancelado por los costos y gastos necesarios para la construcción del bien inmueble (Decreto 1372, 1992).

A diferencia de los servicios gravados que involucran utilización de inmuebles las deducciones son directamente proporcionales a la utilidad.

\section{Resultados}

El resultado de las utilidades de las empresas de Dragado e Ingeniería al realizar un estimado durante el año 2014 al calcular el Impuesto sobre las ventas bajo la modalidad del AIU fue de $\$$ 115.943.224 Ver cuadro 1.3 y el IVA pagado fue de $\$ 178.885 .124$. Ver cuadro 1.4

Cuadro 1.3. Estado de resultado

AGLALA ISNN 2215-7360

2015; 6 (1): 229-251 
ESTADO DE RESULTADOS

Periodo comprendido entre el 1 de Enero y el 31 de Diciembre del 2013

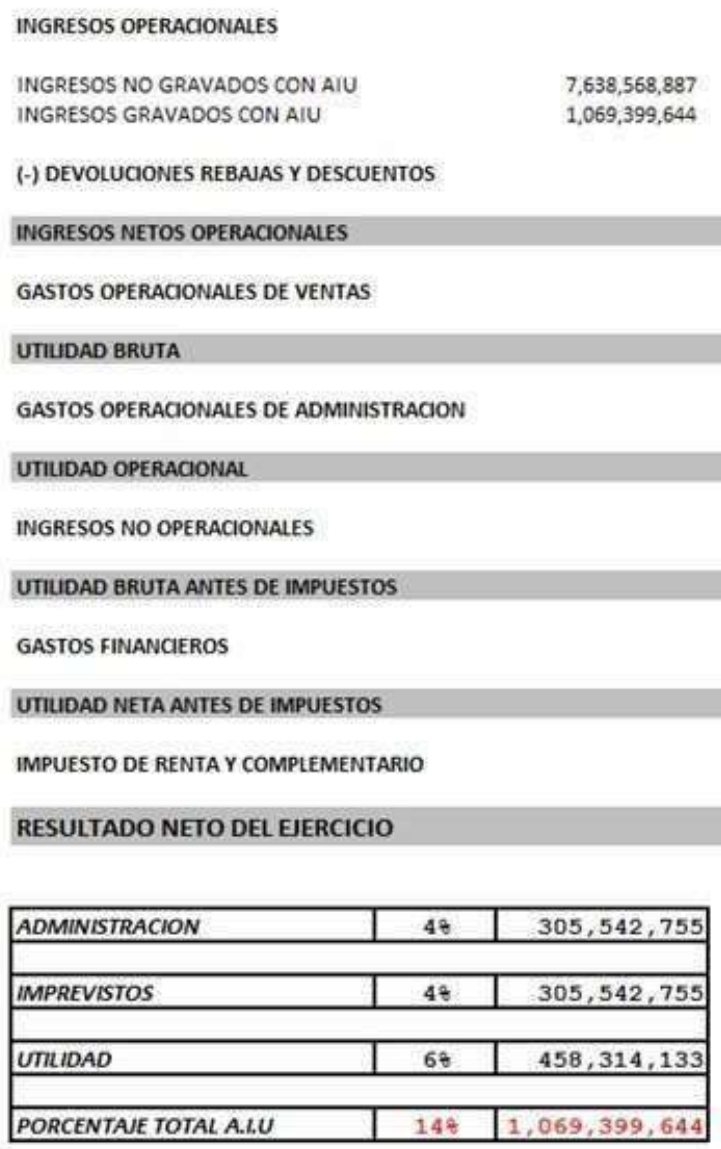

$8,707,968,532$ 8.707 .968 .532

$8,168,109,344$ $539,859,188$ $149,666,593$ $390,192,595$

$390.192,595$

$154,024,371$ $236,168,224$ $120,225,000$ $115,943,224$

Fuente: Elaboración propia

Cuadro 1.4. Liquidación de IVA

AGLALA ISNN 2215-7360

2015; 6 (1): 229-251 


\begin{tabular}{|c|c|}
\hline \multicolumn{2}{|c|}{$\begin{array}{l}\text { SIMULACION DE UQUIDACION DE IVA } \\
\text { SOBRE LOS INGRESOS TOTALES }\end{array}$} \\
\hline \multicolumn{2}{|c|}{ UQQUIDACION DE IVA } \\
\hline INGRESOS GRAVADOS & 8.707 .968 .532 \\
\hline TOTAL INGRESOS & 8.707 .968 .532 \\
\hline TOTAL IVA & 1.393 .274 .965 \\
\hline IVA GENERADO & 1.393 .274 .965 \\
\hline IVA DESCONTABLE REPUESTOS & 103.536 .219 \\
\hline DESCONTABLE SERVICIOS & 36.514 .165 \\
\hline DESCONTABLE HONORARIOS & 9.977 .441 \\
\hline DESCONTABLE ARRIENDO & 79.202 .190 \\
\hline DESCONTABLE NOTARIAS & 310.015 \\
\hline DESCONTABLE SEGUROS & 6.120 .553 \\
\hline DESCONTABLE REGIMEN SIMPLIFICADO & 7.781 .181 \\
\hline TOTAL IVA DESCONTABLE & 243.441 .764 \\
\hline IVA X PAGAR & 1.149 .833 .201 \\
\hline
\end{tabular}

Fuente: Elaboración propia

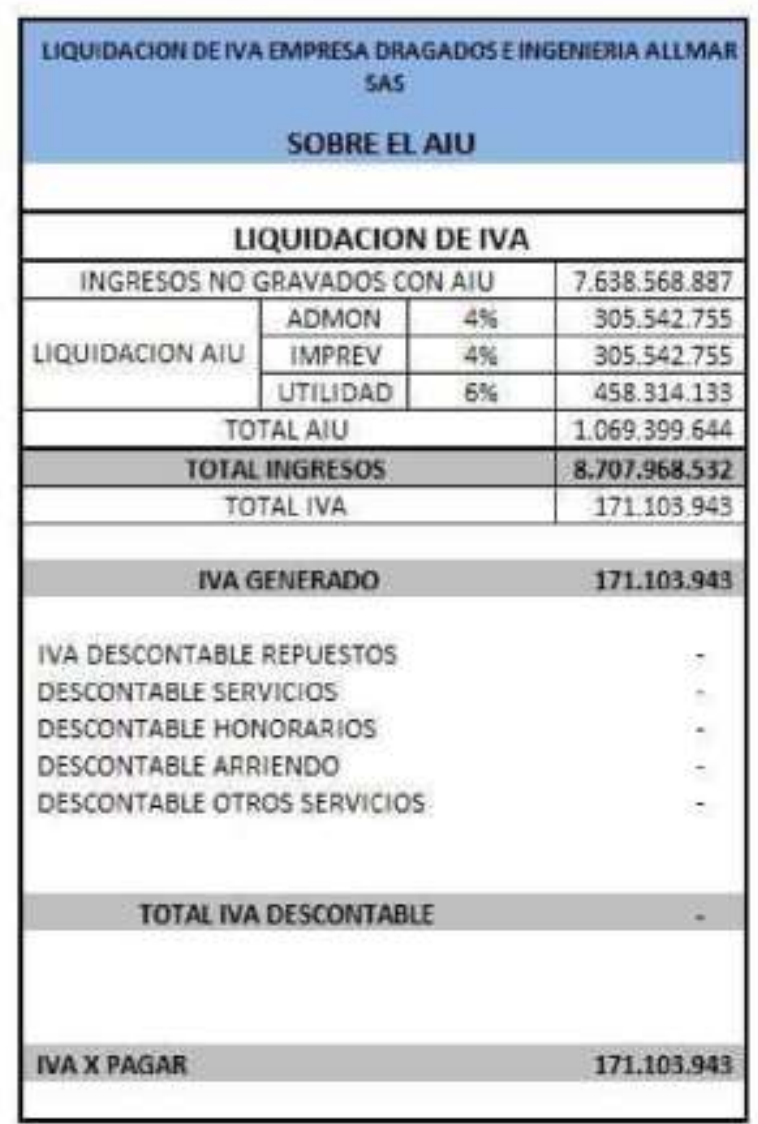

La incidencia de la liquidación del impuesto sobre las ventas bajo la modalidad del AIU en la utilidad de la empresa Dragados e Ingeniería ALLMAR SAS, es que dicha utilidad se vio disminuida en la medida que el porcentaje del IVA descontable bajo esta modalidad solo se puede descontar la proporción correspondiente al AIU, estos serían los gastos de papelería, servicios públicos, entre otros. Por lo tanto, el IVA de los demás costos y gasto se lleva como mayor valor, lo que genera una disminución en la utilidad de la empresa como lo muestra el estado de resultados. Sin embargo la empresa durante al año 2014 no imputo ningún valor al 
impuesto descontable lo que genera un mayor valor a pagar en IVA, por lo tanto al realizar las dos liquidaciones la primera como lo realizó la empresa durante el año gravable 2014 y la segunda cuanto seria el valor a descontar en el IVA según la norma, se puede identificar la diferencia del valor a pagar.

Al determinar el resultado de las utilidades en la sociedad Dragados e Ingeniería ALLMAR SAS durante el año 2014, simulando el cálculo del impuesto sobre las ventas con base a los ingresos totales arrojo una utilidad de $\$ 359.384 .988$ Ver cuadro 1.5 dando como resultado un valor a pagar en IVA de $\$$ 1.149.833.201, valor que es excesivamente alto. Ver cuadro 1.6

Cuadro 1.5. Estados de resultados simulados

AGLALA ISNN 2215-7360

2015; 6 (1): 229-251 


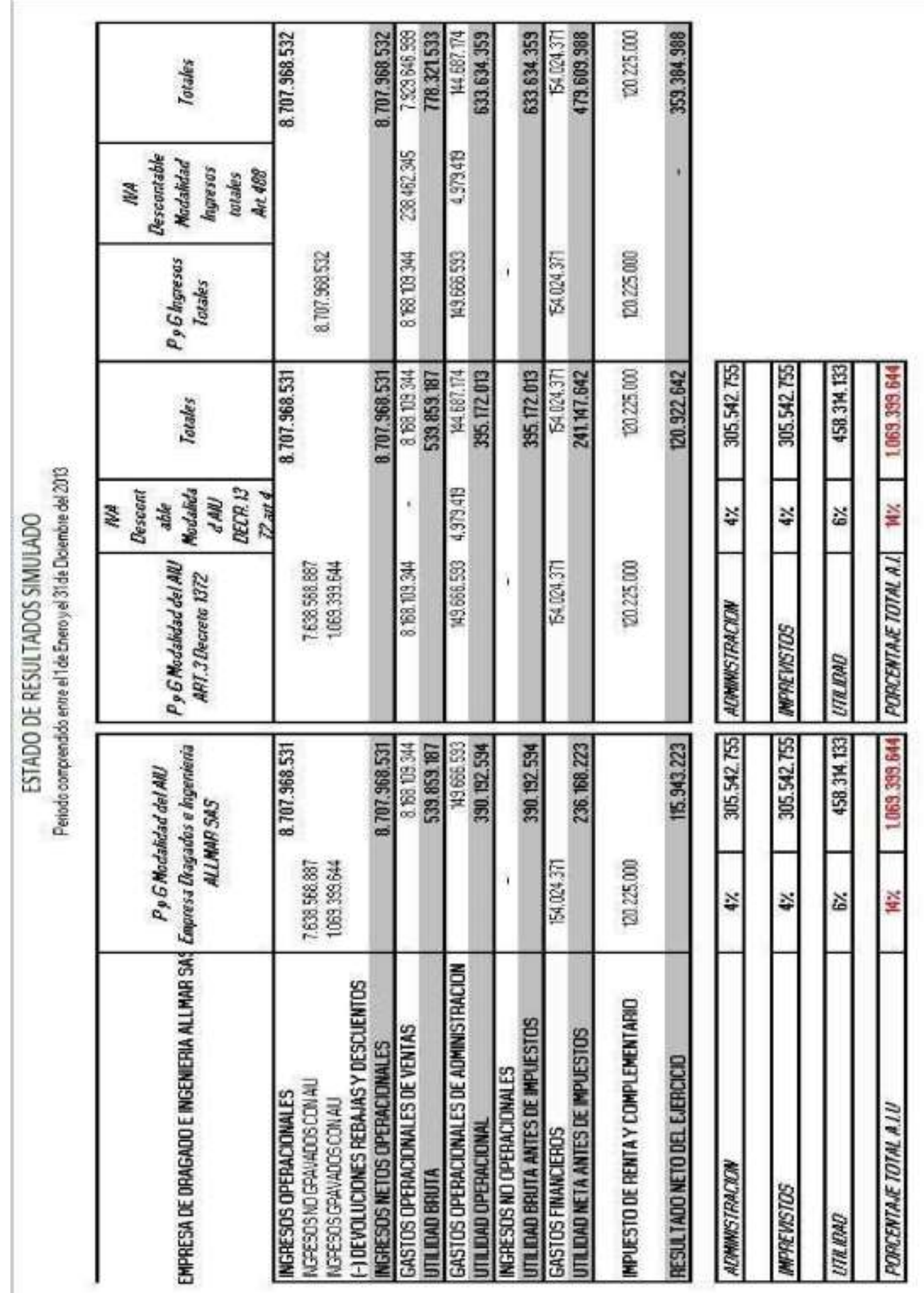

AGLALA ISNN 2215-7360

2015; 6 (1): 229-251 
Fuente: Elaboración propia

Cuadro 1.6. Simulación de liquidación de IVA sobre los ingresos totales

\begin{tabular}{|c|c|}
\hline \multicolumn{2}{|c|}{$\begin{array}{l}\text { SIMULACION DE LIQUIDACION DE IVA } \\
\text { SOBRE LOS INGRESOS TOTALES }\end{array}$} \\
\hline \multicolumn{2}{|c|}{ LIQUIDACION DE IVA } \\
\hline INGRESOS GRAVADOS & $8,707,968,532$ \\
\hline TOTAL INGRESOS & $8,707,968,532$ \\
\hline TOTALIVA & $1,393,274,965$ \\
\hline IVA GENERADO & $1,393,274,965$ \\
\hline IVA DESCONTABLE REPUESTOS & $103,536,219$ \\
\hline DESCONTABLE SERVICIOS & $36,514,165$ \\
\hline DESCONTABLE HONORARIOS & $9,977,441$ \\
\hline DESCONTABLE ARRIENDO & $79,202,190$ \\
\hline DESCONTABLE NOTARIAS & 310,015 \\
\hline DESCONTABLE SEGUROS & $6,120,553$ \\
\hline DESCONTABLE REGIMEN SIMPLIFICADO & $7,781,181$ \\
\hline TOTAL IVA DESCONTABLE & $243,441,764$ \\
\hline IVAX PAGAR & $1,149,833,201$ \\
\hline
\end{tabular}

Fuente:

Elaboración propia

La incidencia de la liquidación del impuesto sobre las ventas bajo la modalidad de los ingresos totales, sobre la utilidad de la empresa es que dicha utilidad se vio aumentada en la medida que el porcentaje del IVA descontable bajo esta modalidad se puede descontar todo lo que represente costo y gasto, lo que genera un aumento en la utilidad de la empresa como lo muestra el estado de resultado.

AGLALA ISNN 2215-7360

2015; 6 (1): 229-251 
Al comparar los dos estados de resultado se puede observar que a pesar de que en el método de la liquidación del IVA sobre los ingresos totales aumenta la utilidad también como contra partida genera un IVA desproporcionado en comparación con la liquidación del IVA con el método del AIU, en el cual se ve disminuida la utilidad, pero a su vez disminuye el impuesto recaudado. Ver Cuadro 1.7

Cuadro 1.7. Simulación y liquidación 


\begin{tabular}{|c|c|}
\hline \multicolumn{2}{|c|}{$\begin{array}{l}\text { SIMULACION DE UQUIDACION DE IVA } \\
\text { SOBRE LOS INGRESOS TOTALES }\end{array}$} \\
\hline \multicolumn{2}{|c|}{ LUQUIDACION DE IVA } \\
\hline INGRE50S GRAVADOS & 8.707 .968 .532 \\
\hline TOTAL INGRESOS & 8.707 .968 .532 \\
\hline TOTAL IVA & 1. 393.274 .965 \\
\hline IVA GENERADO & 1.393 .274 .965 \\
\hline IVA DESCONTASLE REPUESTOS & $103 \$ 36.219$ \\
\hline DESCONTAELE SERVICIOS & $\$ 6.514 .165$ \\
\hline DESCONTAELE HONORARIOS & 9.977 .441 \\
\hline DESCONTAELE ARRIENDO & 79.202 .190 \\
\hline DESCONTABLE NOTARLAS & 310.015 \\
\hline DESCONTABLE SEGUROS & 6.120 .553 \\
\hline DESCONTAELE REGIMAN SIMPUFICADO & 7.781 .181 \\
\hline TOTAL NA DESCONTABLE & 243.441 .754 \\
\hline INAXPAGAR & 1.149.833.201 \\
\hline
\end{tabular}

Fuente: Elaboración propia

\begin{tabular}{|c|c|c|}
\hline \multicolumn{3}{|c|}{$\begin{array}{l}\text { UQQUIDACION DE IVA EMPRESA DRAGADOS EIMGEEIFPIA } \\
\text { ALLMARSAS } \\
\text { SOBRE EL AIU }\end{array}$} \\
\hline \multicolumn{3}{|c|}{ UQUIDACION DE IVA } \\
\hline \multicolumn{2}{|c|}{ INGRESOS NO GRAVADOS CONAIU } & 7638.568 .887 \\
\hline \multirow{3}{*}{ LIQUIDACION AIU } & $\begin{array}{lll}\text { AOMON } & 485 \\
\end{array}$ & 305.542 .755 \\
\hline & \begin{tabular}{l|l} 
IMPAEV & 45 \\
\end{tabular} & 305.542755 \\
\hline & $\begin{array}{ll}\text { UTILIOAD } & \text { 6K } \\
\end{array}$ & 458.314 .133 \\
\hline \multicolumn{2}{|c|}{ TOTALALU } & 1069.399 .644 \\
\hline \multicolumn{2}{|c|}{ TOTAL INGEESOS } & 8.707 .968 .532 \\
\hline \multicolumn{2}{|c|}{ TOTAL IVA } & 271.103 .943 \\
\hline \multicolumn{2}{|c|}{ IVA GENERADO } & 171.103 .943 \\
\hline \multirow{5}{*}{\multicolumn{2}{|c|}{$\begin{array}{l}\text { IVA DESCONTABLE REPUESTOS } \\
\text { DESCONTABLE SERVICIOS } \\
\text { DESCONTABLE HONORARIOS } \\
\text { DESCONTABLE ARRIENDO } \\
\text { DESCONTABLF OTROS SERVICIOS }\end{array}$}} & - \\
\hline & & - \\
\hline & & - \\
\hline & & $=$ \\
\hline & & - \\
\hline \multicolumn{2}{|c|}{ TOTAL TVA DESCONTABLE } & - \\
\hline NAXPAGAR & & 171.103 .943 \\
\hline
\end{tabular}

\begin{tabular}{|c|c|c|}
\hline \multicolumn{3}{|c|}{$\begin{array}{l}\text { UQUIDACON DE NA SIMULADO } \\
\text { SOBRE EL AIU }\end{array}$} \\
\hline \multicolumn{3}{|c|}{ UUQUIDACION DE IVA } \\
\hline \multicolumn{2}{|c|}{ INGRESOS NO GRAVADOS CON AIU } & 7.638 .568 .887 \\
\hline \multirow{3}{*}{ LUQUDACION AIU } & \begin{tabular}{|l|l|} 
AOMON & 48 \\
\end{tabular} & 305542.755 \\
\hline & IMPREV & 305.542 .755 \\
\hline & \begin{tabular}{l|l} 
UTIUDAD & $6:$ \\
\end{tabular} & 458.314 .133 \\
\hline \multicolumn{2}{|c|}{ TOTALAIU } & 1.069 .399 .644 \\
\hline \multicolumn{2}{|c|}{ TOTAL BUGRESOS } & 305.542 .755 \\
\hline \multicolumn{2}{|c|}{ TOTAL IVA } & 171.103 .943 \\
\hline \multicolumn{2}{|c|}{ NA GENERADO } & 171.103 .943 \\
\hline \multicolumn{2}{|c|}{ IVA DESCONTABLE REPUESTOS } & - \\
\hline \multicolumn{2}{|c|}{ DESCONTABLE SERVICIOS } & 1.809924 \\
\hline \multicolumn{2}{|c|}{ DESCONTABLE HONORARIOS } & 2.602 .659 \\
\hline \multicolumn{2}{|c|}{ DESCONITABLE ARRIENDO } & - \\
\hline \multicolumn{2}{|c|}{ DESCONTABLE OTROS SEAVICIOS } & 345.578 \\
\hline \multicolumn{2}{|c|}{ DESCONTABLE LEGALES } & 221.258 \\
\hline \multicolumn{2}{|c|}{ TOTAL NA DESCONTABLE } & $4.979,419$ \\
\hline IVAXPAGAR & & 166.124524 \\
\hline
\end{tabular}

La incidencia al comparar las dos modalidades de liquidación en la empresa Dragados e Ingeniería ALLMAR SAS, se pudo determinar que bajo la modalidad del AIU la empresa disminuye su utilidad debido que el IVA descontable se contabiliza como mayor valor del gasto; y en la modalidad de los ingresos totales la utilidad aumenta puesto que en esta modalidad el impuesto descontable es todo lo que represente costo o gasto de una actividad gravada por lo tanto representa una disminución de los gastos de la empresa. Por otra parte se realizó un simulación de cómo debe aplicarse el IVA descontable según la norma, para que la empresa no solo pueda recuperar un poco de ese impuesto pagado sino que también se vea reflejada esa operación en las utilidades de esta empresa lo que nos generó un aumento de la utilidad de \$4.979.419 correspondiente al impuesto descontable a la parte del AIU de la empresa. 
Al realizar las dos liquidaciones que establece la norma en materia de generar el impuesto a cargo en este tipo de empresas y verificar el desarrollo ordinario de sus operaciones el método que debe aplicar esta empresa es el mismo que siempre han utilizado es decir el método del AIU en la medida que esta empresa no solo se dedica a rellenar y limpiar agua sino que también dentro de sus actividades de Dragado aplican construcción marítima y obras de ingeniería por lo tanto esta empresa no solo debe aplicar el método del AIU sino que también debe imputar el porcentaje de impuesto descontable según lo correspondiente a la utilidad del constructor.

La incidencia que tiene la liquidación del método del AIU con respecto a la utilidad de la empresa, corresponde a una disminución de dicha utilidad, pero este no solo es el método que la norma establece y la que deben aplicar las empresas con relación a sus actividades; sino que también al momento de realizar una licitación o un concurso y ser adjudicado es el más conveniente, esto debido a que la persona que paga el IVA es el contratante por lo tanto la carga impositiva se ve aliviada por este método, sin embargo si se realiza la liquidación del impuesto bajo la modalidad del total de los ingresos la carga impositiva sería demasiado grande y el contratante debería buscar otras alternativas que le serían mucho más económicas, lo que implicaría que la empresa perdería competitividad con respecto a las empresas que participan en este sector.

\section{Conclusión}

Al realizar el análisis y definir las actividades de la empresa Dragados e Ingeniería ALLMAR SAS con respecto al artículo 3 y 8 decreto 1372 se identificó que la entidad dentro de la generación de sus ingresos aplicaron la modalidad del AIU en la liquidación del impuesto

AGLALA ISNN 2215-7360

2015; 6 (1): 229-251 
sobre las ventas del año 2013, modalidad que la norma establece y es la más favorable teniendo en cuenta que al liquidar el impuesto sobre los ingresos totales el impacto sobre la utilidad es beneficioso en la medida que al imputar el IVA descontable disminuye la carga de los gastos, por tanto al aplicar el IVA sobre esta modalidad no solo disminuye la rentabilidad frente a la competencia si no que a su vez correría el riesgo de perder competitividad porque el valor a pagar en IVA es demasiado alto. Adicional cabe resaltar que la empresa durante el año al realizar la liquidación de Impuesto al Valor Agregado IVA bajo la modalidad del AIU no aplicó el impuesto descontable lo que representó una utilidad disminuida, por lo tanto recomendamos a la empresa aplique el IVA descontable, operación que es permitida por la norma y que sería de mucho beneficio para la empresa debido a que al realizar esta operación tendría el beneficio de recuperar el IVA pagado sobre el AIU y también tendría un aumento en sus utilidades.

\section{Bibliografía}

Bravo, Y. (2003). Los beneficios tributarios del impuesto sobre la renta, Julio. Oficina de Estudios Económicos. Recuperado de http://www.dian.gov.co/.

Constitución Política de Colombia (1991).

Correa, R. (2002). Reformas estructurales y crecimiento en América Latina: un análisis de sensibilidad. Revista de la CEPAL, 76. pp. 89-107.

DIAN (2010). Cultura de la Contribución. Disponible en: http://www.dian.gov.co/. DIAN (2005). Documento CEDE 2005, Impuestos y Reformas Tributarias en Colombia. 1980- 
2003. Colombia.

Díaz, F. y Varela, R. (2003). Junquera. Modelos organizativos de las administraciones tributarias y estrategias de las organizaciones avanzadas. España, Instituto de Estudios Fiscales.

Haindl, E. (2005). Colombia Impacto de las reformas tributarias de 2002 y 2003. Informe Final.

Ministerio de Comunicaciones (2008). Modelo Único de Ingreso Servicio y Control Automatizado (MUISCA). Disponible en: http://www.dian.gov.com.

Moller, L. C. (2012). Política Fiscal en Colombia: aprovechamiento de su potencial para lograr una sociedad más equitativa. Bogotá, D.C.: Fedesarrollo, Banco Mundial \& Fundación Adenauer.

Monsalve, R. (2008). Impuestos 2008. Estatuto Tributario Concordado. Medellín, Colombia. Centro Interamericano jurídico - Financiero.

Pérez, Jacobo (2004). Derecho constitucional colombiano, Bogotá, Colombia: Edit. Temis.

Steiner, R. y Medellín J. (2014). Perspectivas Fiscales 2014-2018: Elementos para una nиеva $\begin{array}{lll}\text { Reforma Tributaria. } & \text { Recuperado de }\end{array}$ http://www.fedesarrollo.org.co/wpcontent/uploads/2011/08/CUADERNOSDEFEDES ARROLLO-No.-52-Perspectivas-fiscales-20142018debate_pres_2014_cuad52.pdf. 